\title{
Non-Hodgkin's Lymphoma of the Knee:A Case Report
}

\author{
Ryan Ka Lok Lee ${ }^{1, *}$; James Francis Griffith ${ }^{1}$; Alex Wing Hung Ng ${ }^{1}$; Hillary Ka Ying Tam ${ }^{2}$; \\ Anthony Wing Hung Chan ${ }^{3}$ \\ ${ }^{1}$ Department of Imaging and Interventional Radiology, Prince of Wales Hospital, The Chinese University of Hong Kong, Hong Kong, China \\ 2 Department of Radiology, North District Hospital, Hong Kong, China \\ 3 Department of Pathology, Prince of Wales Hospital, The Chinese University of Hong Kong, Hong Kong, China \\ ${ }^{*}$ Corresponding author: Ryan Ka Lok Lee, Department of Imaging and Interventional Radiology, Prince of Wales Hospital, The Chinese University of Hong Kong, Hong Kong, China. \\ Tel:+85-226321247, E-mail: leekalok2909@yahoo.com.hk
}

Received: August 3, 2012; Revised: October 28, 2012; Accepted: December 29, 2012

\begin{abstract}
Primary musculoskeletal lymphoma presenting as monoarthritis is very rare. Less than 20 cases have been reported. The ultrasound appearances have not been reported to date. We present a young female of primary knee lymphoma with synovial involvement presenting as monoarthritis. The ultrasound and MRI features are discussed.
\end{abstract}

Keywords: Non-Hodgkin's Lymphoma; Knee; Joint

\section{Introduction}

Primary non-Hodgkin lymphoma (NHL) is an uncommon musculoskeletal malignancy, comprising only $3 \%$ of all bone tumors (1). Synovial involvement by lymphoma is extremely rare (1). Intra-articular lymphoma has nonspecific clinical and imaging appearances. However, the presence of regional lymphadenopathy and focal synovial proliferation should raise suspicion of this rare diagnosis. This case report also shows the ultrasound appearances of intra-articular lymphoma that have not been previously reported.

\section{Case Presentation}

A 27-year-old Indonesian female presented with progressive knee swelling and pain for a month after a minor sprain. She had previously enjoyed good past health. She had no other joint symptoms or any systemic symptoms such as weight loss, fever and night sweats. Physical examination revealed moderate knee joint swelling with joint effusion. The knee could not be fully extended. No redness or increase in temperature of the knee joint was present. There was tenderness at the medial joint line. All the inflammatory blood markers were negative. Knee joint fluid aspiration yielded slightly turbid fluid with a small number of lymphocytes and a negative Gram stain and bacterial culture. Knee radiography revealed moderate soft tissue thickening in the medial aspect of the knee with mild to moderate osteolysis of the medial femoral condyles and moderate resorption of the medial condylar cortex (Figure 1). Ultrasound of the knee showed a small to moderate-sized joint effusion with severe synovial thick- ening at the anteromedial aspect of the knee, mainly at the medial patellofemoral recess and the medial side of the knee joint (Figure $2 \mathrm{~A}$ ). Moderate cortical irregularity of the medial femoral condyle was present (Figure $2 \mathrm{~B}$ ). Only very mild vascularity was present within this area of synovial thickening. MRI showed a large soft tissue lesion present on the medial side of the knee joint (Figure 3 A), involving the synovium and adjacent medial femoral condyle with moderate periosteal elevation. This lesion extended deep to the medial collateral ligament and extended into the intercondylar notch posteriorly encompassing the femoral attachment of the medial belly of gastrocnemius muscle. The soft tissue mass was slightly T1-hypointense and T2-hyperintense to skeletal muscle (Figure $3 \mathrm{~A}, 3 \mathrm{~B}$ ). No evidence of hemosiderin was present. There were two moderately enlarged popliteal lymph nodes present (Figure 4). In addition, abnormal bone marrow signal (low in $\mathrm{T} 1$ and heterogeneously high in $\mathrm{T} 2$ FSE) was present in the distal femur consistent with marrow involvement or infiltration (Figure $3 \mathrm{~A}, 3 \mathrm{~B}$ ). Patient refused gadolinium contrast injection. Ultrasound guided biopsy of this synovial thickening/soft tissue revealed fibrous tissue infiltrated by diffuse sheets of atypical lymphoid cells (Figure 5). Immunohistochemical staining of these atypical large lymphoid cells revealed positivity for lymphoid marker (LCA), B cell marker (CD20) and bcl-2. Proliferation index by Ki67 (MIB-1 immunostaining) was estimated as 95\%. Features were compatible with diffuse large B-cell non Hodgkin lymphoma (NHL). The patient refused treatment B-cell NHL and further investigation (including abdominopelvic CT scan). However, physical

Copyright ( ) 2015, Tehran University of Medical Sciences and Iranian Society of Radiology. This is an open-access article distributed under the terms of the Creative Commons Attribution-NonCommercial 4.0 International License (http://creativecommons.org/licenses/by-nc/4.0/) which permits copy and redistribute the material just in noncommercial usages, provided the original work is properly cited. 
examination did not reveal enlarged systemic lymph nodes or hepatosplenomegaly. In view of clinical absence of systemic involvement, this was considered to be primary lymphoma of the knee. There was no clinical or other evidence that the patient was immunocompromised.

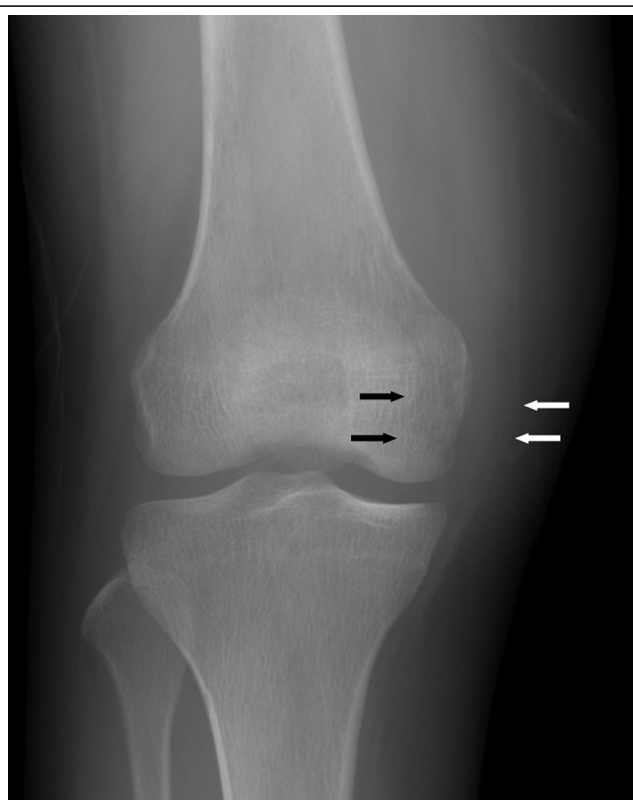

Figure 1. A 27-year-old female presented with progressive knee swelling and pain for one month. Anteroposterior radiography of the knee shows moderate soft tissue thickening of the medial aspect of the knee (white arrows) with mild to moderate osteolysis of the medial femoral condyle (black arrows) and moderate resorption of medial condylar cortex.
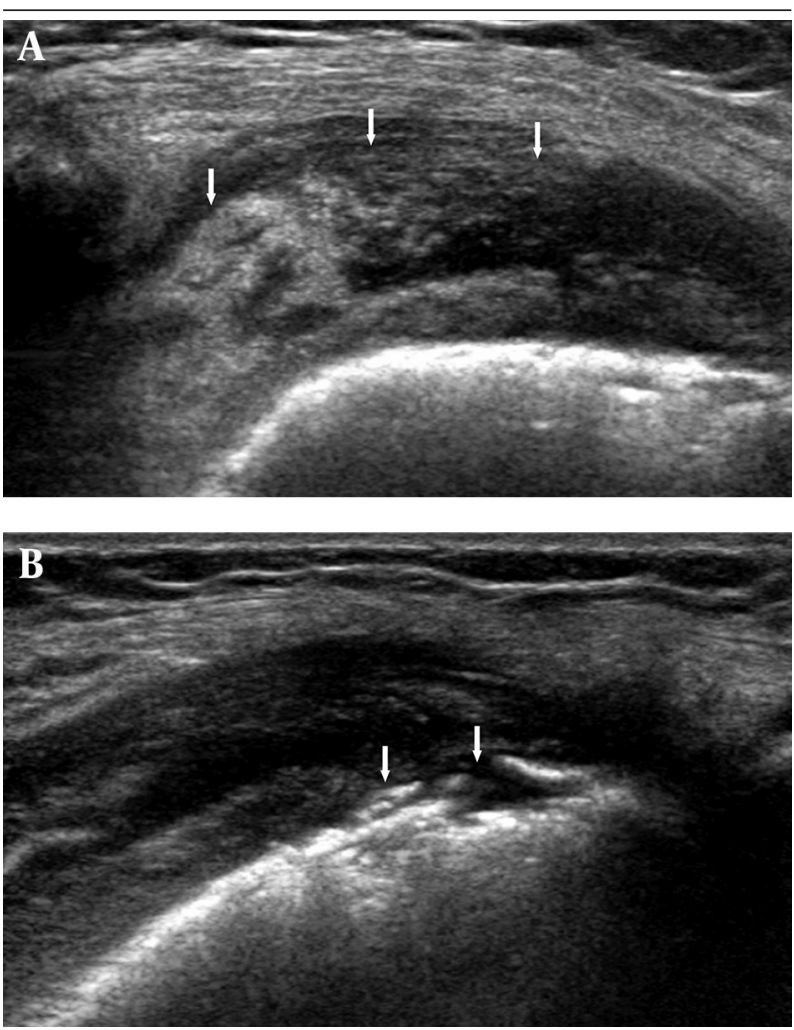

Figure 2. A, Oblique transverse ultrasound of the medial patellofemora recess. There is severe synovial thickening on the medial aspect of the knee joint. B, Transverse oblique ultrasound of the distal medial femoral condyle. There is moderate cortical irregularity (white arrows) of the femoral condyle adjacent to the synovial thickening.
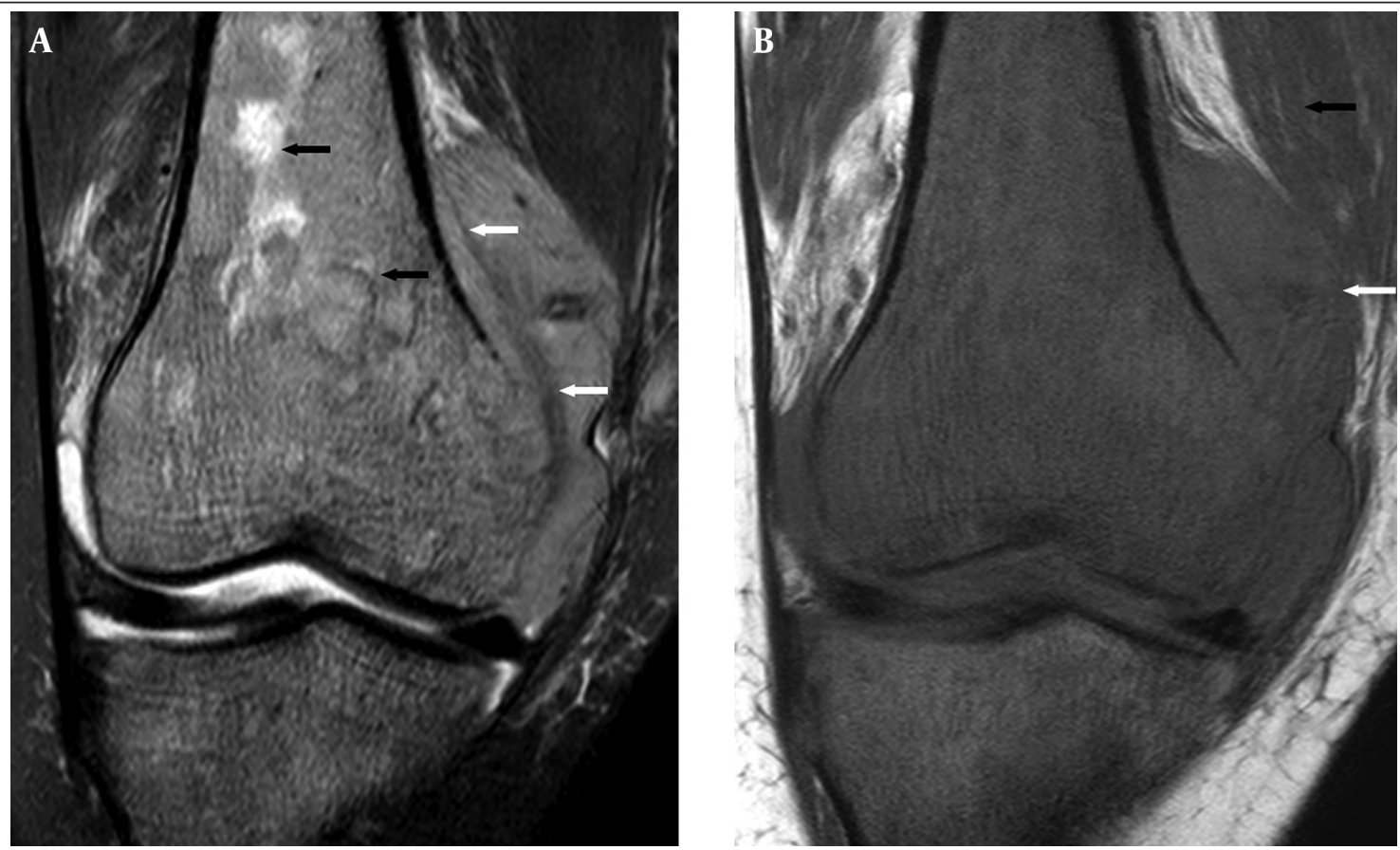

Figure 3. A, Coronal T2-weighted fat suppressed MRI of the knee. There is a large soft tissue lesion within the medial aspect of the knee joint; the lesion predominantly involves the synovium and adjacent medial femoral condyle with moderate periosteal elevation (white arrows). The synovial thickening is T2- hyperintense compared to the skeletal muscle. There is also abnormal T2- hyperintensity of the bone marrow in the distal femur (black arrows). $\mathrm{B}$, Coronal T1-weighted MRI of the knee. The lesion (white arrow) is T1- isointense compared to skeletal muscle (black arrow); there is also abnormal low bone marrow signal in this T1-weighted sequence. 
Lee RKL et al.

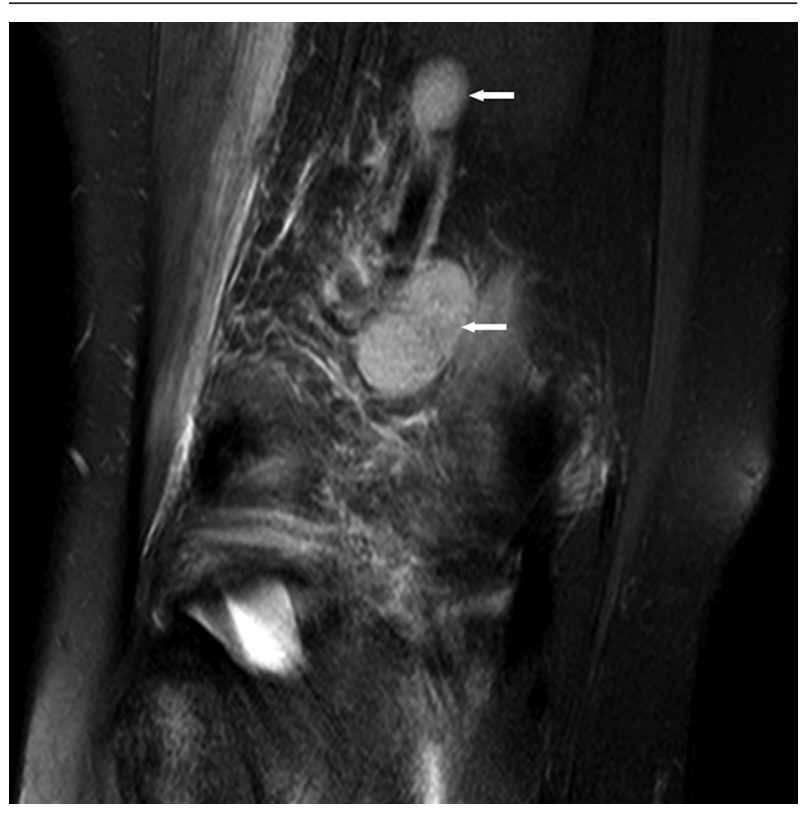

Figure 4. Coronal T2-weighted fat-suppressed MRI of the knee; there are two moderately enlarged lymph nodes (white arrows) present in the popliteal fossa.

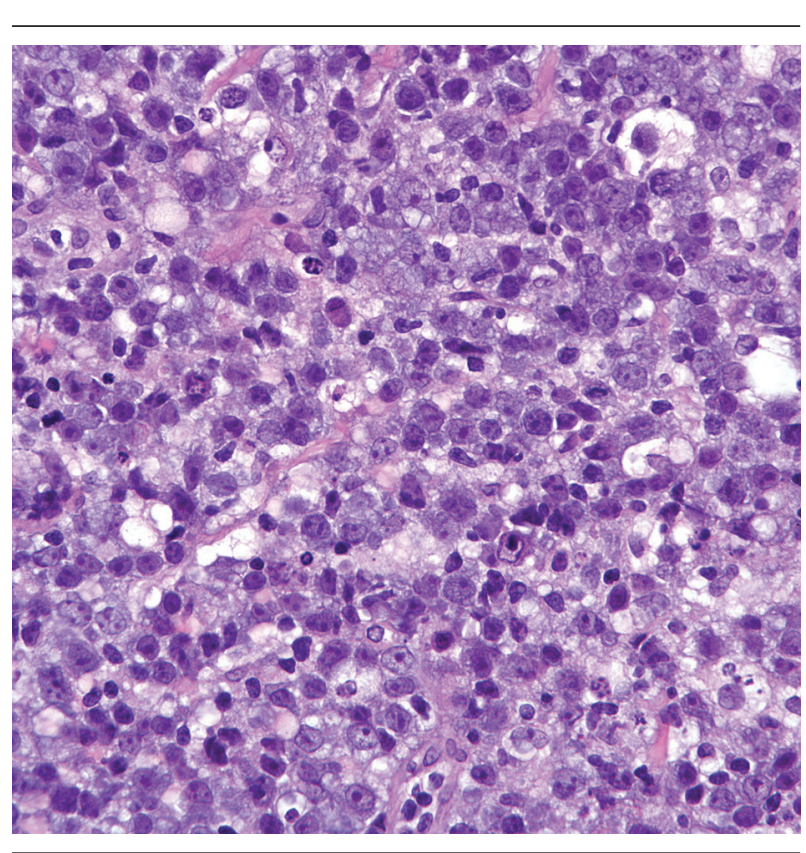

Figure 5. Histological slide of synovium in hematoxylin and eosin stain (magnification $\times 400$ ) shows diffuse sheets of atypical large-sized lymphoid cells with vesicular nuclei, dispersed chromatin and prominent nucleoli, suggestive of lymphoma.

\section{Discussion}

Musculoskeletal involvement affects about 7\% of NHL patients, typically as metastatic disease and infrequently as a primary lymphoma of either the bone or soft tissues (2-4). Presentation with joint involvement is very rare (2). To the best of our knowledge, there are only 16 cases of joint involvement with synovial infiltration reported in English literatures (1-4), most of which had known pre-existing NHL $(1,2)$. Of the reported cases, males and females were equally affected with a wide age range of 13 to 76 years old (1). The knee joint (11 cases) is the most commonly involved joint followed by the elbow (two cases), sternoclavicular joint (one case), shoulder (one case) and wrist (one case) (1). Joint involvement in NHL usually presents with symptoms suggestive of an inflammatory arthropathy or low grade infection such as joint pain, swelling and limitation of movement affecting a single joint rather than multiple joints $(2,5,6)$. A minority of patients also has systemic symptoms such as weight loss, night sweats and fever $(2,7)$. About one-third of all cases are immunocompromised (8-10). Serological tests are usually non-contributory. Similarly, synovial fluid analysis may not always help in distinguishing joint lymphoma from a low-grade chronic septic arthritis or inflammatory arthropathy since all these conditions can lead to mild synovial leukocytosis with lymphocytic predominance (1). Synovial involvement of lymphoma usually occurs through direct extension from the bone $(1,2)$. In this case, the lymphoma seemed to be primarily located in the medial femoral condyle with secondary infiltration of the synovium on the medial aspect of the knee. In a few of the previous cases of intra-articular lymphoma, the lymphoma seemed to be primarily synovial in origin as there was no bone involvement as judged by MRI (11, 12). In the absence of serial histological sectioning, MRI is the only reliable means to determine whether there is co-existing bone marrow involvement $(11,12)$. Radiography may reveal para-articular bony erosion or osteolysis though in half (8 out of 16 cases) of the reported cases, radiography will be unremarkable except for soft tissue swelling (1). Ultrasound is useful in distinguishing effusion from synovial thickening. Focal synovial thickening as in this case is a useful marker of an infiltrative disorder such as lymphoma as TB and inflammatory arthropathy would usually give rise to more diffuse synovial thickening. Ultrasound also facilitates imaging-guided synovial biopsy. Ultrasound findings of joint lymphoma have not previously been reported. MRI allows a complete assessment of the bone marrow, bone cortex, joint, and lymph node involvement. Similar to all the previous reported cases, the signal intensity of lymphoma in this case is also non-specific ( $\mathrm{T} 1$ Whypointense and $\mathrm{T} 2 \mathrm{~W}$ hyperintense) though the signal characteristics are helpful in distinguishing lymphoma from other synovial disorders such as pigmented villonodular synovitis or synovial osteochondromatosis (13). The presence of enlarged regional lymph nodes is only seen with tuberculous infection, lymphoma, and malignancy, which is helpful in further narrowing the differential diagnosis. Only $2-4 \%$ of musculoskeletal sarcomas have nodal involvement at presentation $(14,15)$. On the other hand, nodal enlargement with musculoskeletal lymphoma is very common $(16,17)$. The differential diagnosis of focal synovial thickening 
includes synovial chondromatosis, pigmented villonodular synovitis (PVNS), focal nodular synovitis, chronic infection such as tuberculosis, crystal or amyloid depositional disease and rarely synovial sarcoma (18). The MR appearances also distinguish most of these entities from one another. Synovial chondromatosis is associated with T1-weighted and T2-weighted hypointense nodules or nodules with a center showing fat signal (osteochondral metaplasia). Pigmented villonodular synovitis (PVNS) is associated with hypointense foci due to hemosiderin deposition on $\mathrm{T} 2^{*}$-weighted sequences. Nodular synovitis will not cause periosteal elevation, medullary infiltration or nodal enlargement as seen in this case. Chronic infection with bone marrow involvement is the main consideration though it is usually associated with more marked peri-articular inflammation and a synovium of higher T2-weighted signal intensity than lymphoma as seen in this case. Gout and amyloid are often hypointense on T2-weighted sequences. Intra-articular synovial sarcoma is rare and may have similar imaging features and clinical presentations. However, it usually presents as a periarticular rather than articular mass and would infiltrate the medullary canal as a late feature (12). As the imaging findings are not pathognomonic, ultrasound-guided synovial biopsy was helpful in confirming the diagnosis of lymphoma. The absence of systemic work-up (such as abdominopelvic CT scan) is the main limitation of this case report as the patient refused further investigation, though clinically there was no systemic adenopathy, hepatosplenomegaly or constitutional symptoms to indicate involvement beyond the knee region. Intra-articular lymphoma with synovial involvement is very rare although it can be broadly distinguished from other synovial diseases by MRI. However, the imaging findings are not entirely specific and image -guided synovial biopsy is required for definitive diagnosis.

\section{References}

1. Donovan A, Schweitzer ME, Garcia RA, Nomikos G. Chronic lymphocytic leukemia/small lymphocytic lymphoma presenting as septic arthritis of the shoulder. Skeletal Radiol. 20 08;37(11):1035-9.
2. Neri R, Giorgetti M, Zampa V, Iacopetti V, Vannucci P, Tognetti A, et al. Case report. Elbow monoarthritis revealing B cell nonHodgkin's lymphoma. Clin Rheumatol. 2013;32 Suppl 1:S111-4.

3. Ruzek KA, Wenger DE. The multiple faces of lymphoma of the musculoskeletal system. Skeletal Radiol. 2004;33(1):1-8.

4. Malloy PC, Fishman EK, Magid D. Lymphoma of bone, muscle, and skin: CT findings. AJR Am J Roentgenol. 1992;159(4):805-9.

5. Jawa A, Lieberman AE, Alexieva CC, Jupiter JB. Primary intra-articular non-Hodgkin's lymphoma of the elbow. A case report. J Bone Joint Surg Am. 2006;88(12):2730-4.

6. Birlik M, Akar S, Onen F, Ozcan MA, Bacakoglu A, Ozkal S, et al. Articular, B-cell, non-Hodgkin's lymphoma mimicking rheumatoid arthritis: synovial involvement in a small hand joint. Rheumatol Int. 2004;24(3):169-72.

7. Daneshpouy M, Bataille D, Rivet J, Riviere O, Morel P, Amouroux J, et al. Peripheral T-cell lymphoma with eosinophilia presenting as monoarthritis: a case study. Leuk Lymphoma. 2002 43(9):1875-9.

8. Goodlad JR, Hollowood K, Smith MA, Chan JK, Fletcher CD. Primary juxtaarticular soft tissue lymphoma arising in the vicinity of inflamed joints in patients with rheumatoid arthritis. Histopathology.1999;34(3):199-204.

9. Khan SY, Hutchinson DG. Primary synovial non-Hodgkin's lymphoma in association with ankylosing spondylitis. Rheumatology (Oxford). 2004;43(3):391.

10. Arredondo J, Worland RL, Sinnenberg RJ, Qureshi GD. NonHodgkin's lymphoma as an unexpected diagnosis in a shoulder arthroplasty. J Arthroplasty. 1999;14(1):108-11.

11. Ogose A, Kawashima H, Hotta T, Endo N, Umezu H, Nomoto N Conditions suggesting lymphoma: case 3. Primary synovial lymphoma with osteoclast-like giant cells presenting as a tenosynovial giant-cell tumor. J Clin Oncol. 2005;23(16):3847-8.

12. Jamieson KA, Beggs I, Robb JE. Synovial presentation of nonHodgkin's lymphoma. Br J Radiol.1998;71(849):980-2.

13. Suresh S, Saifuddin A, O'Donnell P. Lymphoma presenting as a musculoskeletal soft tissue mass: MRI findings in 24 cases. Eur Radiol. 2008;18(11):2628-34.

14. Riad S, Griffin AM, Liberman B, Blackstein ME, Catton CN, Kandel RA, et al. Lymph node metastasis in soft tissue sarcoma in an extremity. Clin Orthop Relat Res. 2004(426):129-34.

15. Fong Y, Coit DG, Woodruff JM, Brennan MF. Lymph node metastasis from soft tissue sarcoma in adults. Analysis of data from a prospective database of 1772 sarcoma patients. Ann Surg. 1993;217(1):72-7.

16. Hwang S. Imaging of lymphoma of the musculoskeletal system Magn Reson Imaging Clin N Am. 2010;18(1):75-93.

17. Damron TA, Le MH, Rooney MT, Vermont A, Poiesz BJ. Lymphoma presenting as a soft tissue mass. A soft tissue sarcoma simulator. Clin Orthop Relat Res.1999(360):221-30.

18. Jaganathan S, Goyal A, Gadodia A, Rastogi S, Mittal R, Gamanagatti S. Spectrum of synovial pathologies: a pictorial assay. Curr Probl Diagn Radiol. 2012;41(1):30-42. 